\title{
Results of Lisfranc's Surgery in Athletic Patients
}

\author{
A. Saxena ${ }^{1}$, L. Shou ${ }^{2}$, M. Fournier ${ }^{3}$ \\ 1 Department of Sports Medicine, Sutter-Palo Alto, El Camino Real, Palo Alto (CA), U.S.A. \\ 2 Reconstructive Orthopedics, Medford (NJ), U.S.A. \\ 3 Gundersen Health System, LaCrosse (WI), U.S.A.
}

\section{CORRESPONDING AUTHOR: \\ Amol Saxena \\ Department of Sports Medicine \\ Sutter-Palo Alto \\ 795 El Camino Real \\ Clark Building \\ Palo Alto (CA) 94301, U.S.A. \\ E-mail: heysax@aol.com}

DOI:

10.32098/mltj.04.2021.17

LEVEL OF EVIDENCE: 4

\begin{abstract}
SUMMARY
Background. Early detection and treatment of Lisfranc injuries in the athletic population is paramount for successful return to sport as well as outcome. The present study evaluates surgical interventions for these types of injuries as well as return to activity (RTA), function and post-operative degenerative joint disease.

Methods. The data from nineteen patients prospectively followed from 2008 through 2016 presented. Of these patients, six $(31.6 \%)$ were treated with suture-button fixation, seven $(36.8 \%)$ with metallic screws and plates and six $(31.6 \%)$ with bioabsorbable screws. The average RTA was $24.0 \pm 10.3$ weeks. The post-operative Roles and Maudsley (RM) score was $1.2 \pm 0.4 .21 \%$ of the patients were noted to have joint space narrowing at the final post-operative $\mathrm{x}$-ray. Hardware removal was performed in six (31.6\%) patients.

Results. All of the patients were able to return to their sporting activities.

Conclusions. Based on the current study, anatomic reduction showed to be the highest predictor of surgical outcome, regardless of fixation technique, consistent with previous studies. ORIF of Lisfranc injuries in the athletic population provides safe and predictable results with good medium-term outcomes.
\end{abstract}

\section{KEY WORDS}

Atblete; Lisfranc joint; return to activity; suture-button.

\section{INTRODUCTION}

The incidence of Lisfranc injuries has increased over the years as participation in sports continues to grow. Approximately 1 in 55,000 people have had a Lisfranc injury reported in the United States on an annual basis (1). These injuries are missed in nearly $20 \%$ initial presentation (2). Despite the lower incidence compared to other foot and ankle pathology, these fractures or dislocations can be devastating injuries due to high incidence of post-traumatic degenerative joint disease.

The anatomy of the Lisfranc joint is very complex and involves the articulations between the bases of the metatarsals with the cuneiforms and cuboid, stabilized by weak dorsal, strong plantar, and interosseous ligaments (3). Lisfranc injuries can occur as a result of a wide variety of traumatic mechanisms and are separated into two groups: high and low energy (4). High-energy Lisfranc injuries often occur due to a direct axial load to the midfoot, frequently seen as a result from a motor vehicle accident or crush injury. These are more likely associated with bony injuries and compartment syndromes (5).
Lisfranc trauma in the athletic population typically occur from indirect mechanisms and can cause more ligamentous than bony injuries (5). They are categorized a low energy trauma and typically caused by an axial longitudinal force on a hyper-plantarflexed foot (6).

Clinical assessment is crucial in early diagnosis of Lisfranc injuries. As previously mentioned, Lisfranc injuries in the athletic population are often purely ligamentous and have subtle presentations (7). Patients may or may not have generalized swelling and/or bruising over the midfoot but usually have pain with manipulation of the tarsometatarsal joints. Initial weight-bearing radiographs should be taken, with comparison contralateral films to help in diagnosis subtle Lisfranc's injury.

Nunley and Vertullo introduced a classification system for subtle, low-energy Lisfranc injuries (8). Stage 1 injuries are classified as a midfoot or Lisfranc sprain and do not show any diastasis on anteroposterior radiograph. Stage 2 injuries have a diastasis between 2 and $5 \mathrm{~mm}$ between the first and second metatarsal bases on anteroposterior radiograph with- 
out collapse of arch height. Stage 3 injuries are represented by greater than $5 \mathrm{~mm}$ diastasis with collapse of arch height. Additional imaging can also be helpful with diagnosis and classification purposes. Bone scans or SPECT (Single Photon Emission Computerized Tomography) scans are used and can show some metabolic bone activity, especially in stage 1 injuries $(9,10)$. CT (Computerized Tomography) scans are useful in assessing fracture comminution. Magnetic Resonance Imaging (MRI) is often used to assess for ligament tears as well as bone marrow edema. Raikin et al. compared MRI results to intra-operative findings and determined that MRI is accurate in detecting traumatic injury to the Lisfranc ligament (11). However, it is important to keep in mind that MRI is not a weight-bearing test, so it may not provide much additional information in subtle cases (9). Many surgical fixation techniques have been described in the literature for Lisfranc fractures and dislocations (10, 12-20). In this prospective study, we analyze the clinical outcomes of athletic patients with Lisfranc injuries who required surgical intervention. Screw and/or plate, suture-button, bioabsorbable pin, and Kirschener-wire fixation were used in this study. It is acknowledged that fixation variability may obscure the results and it is not anticipated we would have definitive findings as to the best fixation type. Medium to long-term follow up is analyzed to assess clinical outcomes including return to sport, maintenance of reduction, and complications.

\section{METHODS}

Active patients under than age of 40 years who underwent open reduction or percutaneous fixation surgical treatment for acute Lisfranc injuries from January 2008- December 2016 were prospectively followed (18). An IRB was obtained from our institution (SHIRB \#: 2017.081EXP IRBNet \#: 1089721-8). During this time frame, 112 patients with Lisfranc injuries were treated by the primary author. Pre-operative imaging included weightbearing xrays, CT or MRI scans if needed to further assess the injury. Inclusion criteria required the patient to be athletically active, ability to document their sport, return to activity timeframe (RTA), radiographic findings (for measurement of reduction and assessment of arthritis) and Roles and Maudsley (RM) scores (21). Exclusion criteria was the inability to document the previous information and loss of follow-up prior to one-year, chronic injuries (past six weeks), as well as those undergoing primary fusion for severe intra-articular injuries.

Nineteen patients of the $112(17 \%)$ met the inclusion criterion, six $(31.6 \%)$ males and $13(68.4 \%)$ females, average age $20.6 \pm 7.9$ years $(r=13-39)$. There were nine "Right" feet and 10 "Left" feet. Sixteen were medial Lisfranc injuries with diastasis of the Lisfranc's articulation and three involved the lateral Lisfranc's region at the level of the $4^{\text {th }}$ metatarsal-cuboid region. All were active in sports, the majority in soccer (seven individuals), two each in volleyball and basketball, four in running sports such as track and field, and one each in football and swimming. The fixation type was recorded. Post-operative $\mathrm{X}$-ray reductions were assessed by an individual (typically a Fellow) not involved with the index surgery, as well as the incidence of post-operative DJD, RM scores and return to activity time frames (15). Reductions were determined to be anatomic if $\leq 1 \mathrm{~mm}$ of offset of the metatarsal to corresponding cuneiform or cuboid or widening $<4 \mathrm{~mm}$ of the region of Lisfranc's ligament (first cuneiform to the second metatarsal or distance between the first and second metatarsal bases) Student-T, one-way ANOVA for post-hoc comparison and Chi-squared tests were used to analyze the data with STATA Version $14.2^{\circledR}$ (Statacorp LLC, College Station TX, USA), with $\mathrm{P}$-value set at $\leq 0.05$.

\section{Surgical technique}

The surgery is typically performed on an outpatient basis using general anesthesia with a regional local block. A thigh or mid-calf tourniquet may be used. The patient is then prepped and draped in the normal aseptic fashion.

\section{Screw fixation}

Three $(16 \%)$ patients had "traditional" one screw fixation. Attention was directed to the medial aspect of the first metatarsal cuneiform joint. A small incision was made over the medial aspect of the first cuneiform. A large reduction clamp was then utilized to reduce the diastasis with the tips placed on the medial cuneiform and lateral aspect of the second or third metatarsal base. A $3.5 \mathrm{~mm}$ cortical screw was then placed across the medial cuneiform and into the base of the second metatarsal, following the trajectory of Lisfranc's ligament. After placement of this screw, the bone clamp was removed. Fluoroscopic guidance was used to confirm reduction and alignment and placement of hardware. Inter-cuneiform instability was then assessed. If instability was confirmed, proximal screw was placed from medial across the cuneiforms to reduce the diastasis. A bridge plate or distal screw was used to stabilize the first metatarsal to the first cuneiform. Plate fixation was used to stabilize lesser metatarsal fractures. The hardware was typically removed after 12 weeks. This was performed for any screw spanning a joint, bridge plating and when prominent. In one patient $(5 \%)$ with open physes, $\mathrm{K}$-wires were used and 
removed at six weeks. Screws placed across joints (i.e., first TMT) were abandoned after 2013 for concern of inducing osteochondral defects and subsequent potential for arthrosis; bridge plating was subsequently used in this situation (16) (figures 1, 2).

\section{Bio-absorbable Screw}

This technique was performed on two $(10.5 \%)$ patients treated from 2008-10 based on prior studies showing reasonable results $(14,17)$. In situations where hardware is to be left remaining, i.e., under a flap after an open injury, bio-screws are an option. Attention is directed to the area of the Lisfranc joint. A bone reduction clamp as above, was carefully used percutaneously to reduce and compress the joint. Alignment and reduction are assessed with fluoroscopic guidance in multiple planes. Then, a $2.5 \mathrm{~mm}$ drill was used across the Lisfranc joint. Subsequently, a $3.5 \mathrm{~mm}$ bio-absorbable screw was placed across the joint. The bone clamp was removed. Stability and alignment were once again checked with fluoroscopic guidance. If the stability was insufficient, the bioscrew was exchanged for a metallic screw.

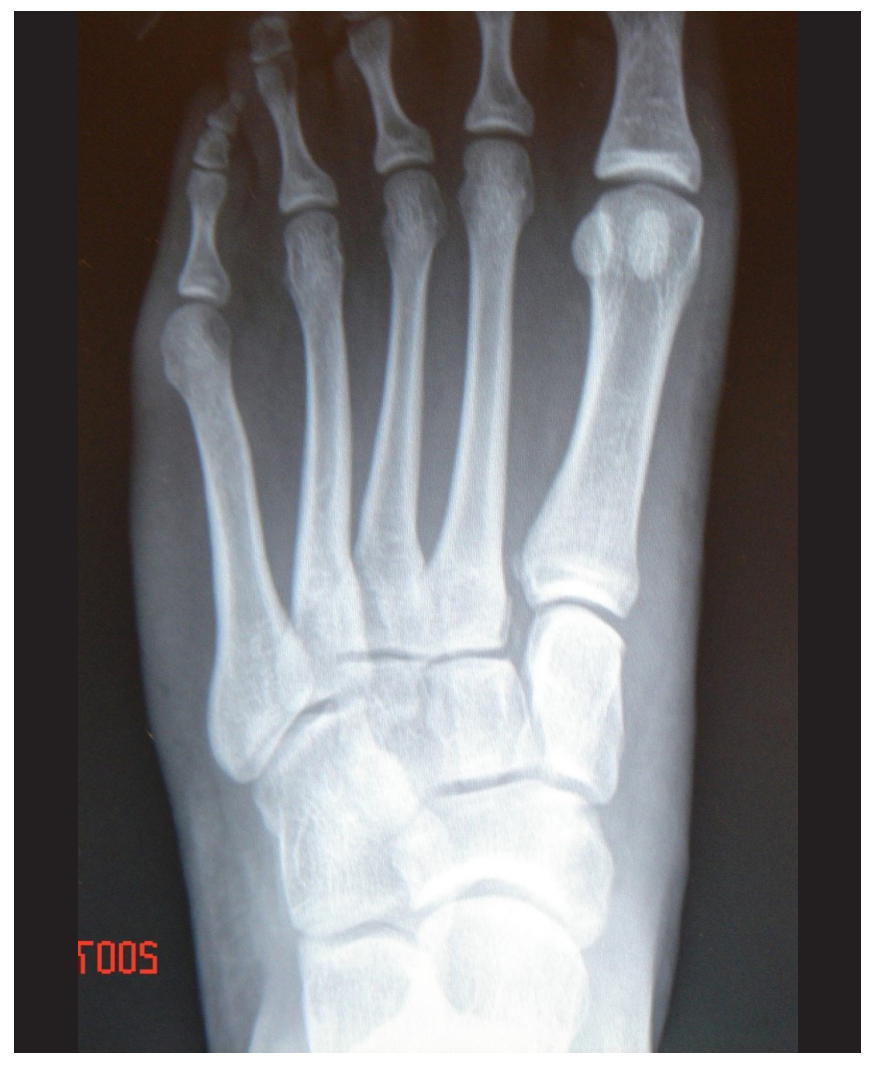

Figure 1. Pre-operative radiograph.

\section{Suture-button}

A contraindication for this technique is ligamentous laxity. To date, there is a small series (seven patients) supporting this technique in dancers and high-level athletes $(10,19)$. Similar to bioscrews, open injuries or in cases when the hardware will remain, suture-button technique may be an option. This was performed on six $(31.6 \%)$ of patients. Attention was directed to the medial aspect of the first metatarsal cuneiform joint. Layered dissection was made carefully taking great care to avoid any vital neurovascular structures. A bone reduction clamp was placed as above to reduce the diastasis. After adequate exposure for the first cuneiform, a drill bit was introduced from medial to lateral across the Lisfranc joint towards the base of the second metatarsal. Fluoroscopic guidance was used in multiple planes to assess proper alignment. A single suture-button device with braided non-absorbable suture and metallic buttons was then inserted through the pre-drilled hole across the Lisfranc joint and tied down medially with adequate compression and maintaining anatomic alignment of the Lisfranc joint. Once again, intra-operative fluoroscopy was used to assess proper seating of the metal-

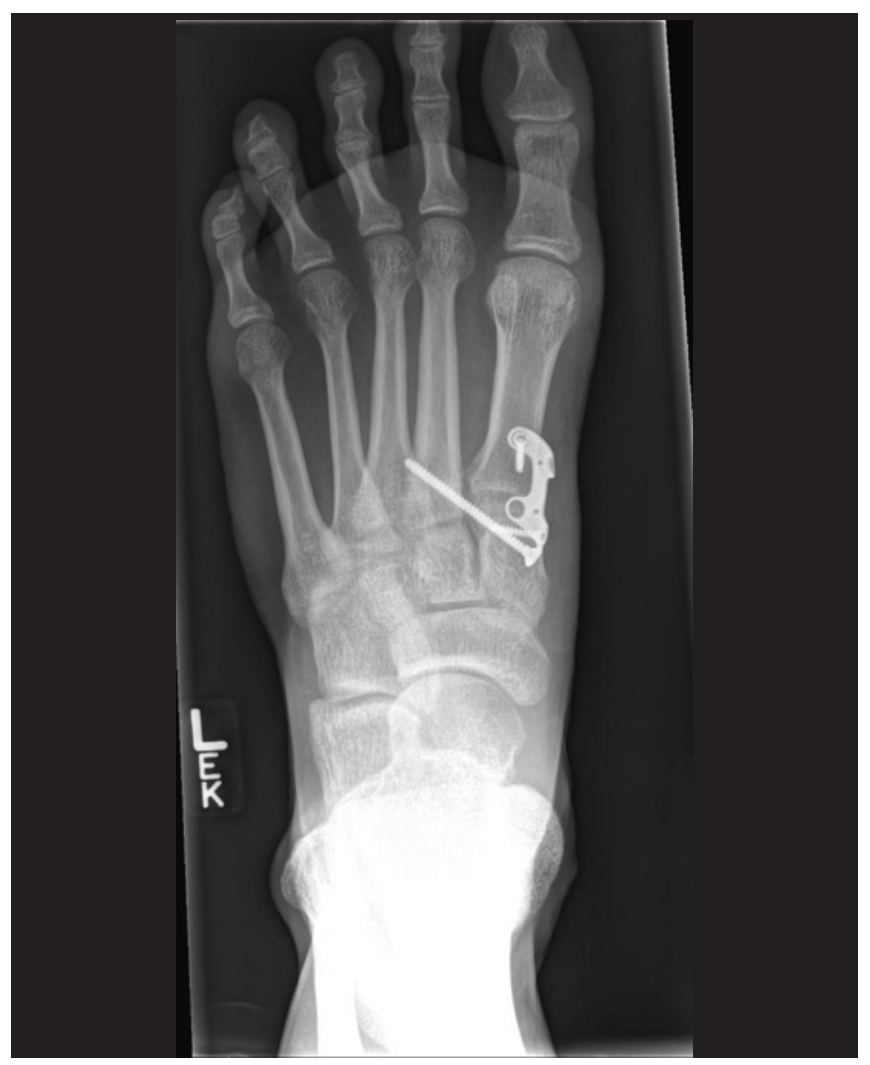

Figure 2. Post operative radiograph.

Muscles, Ligaments and Tendons Journal 2021;11 (4) 
lic suture-buttons, which should be flush to the bone. The bone clamp was released. If stability is insufficient, a metallic screw was place proximal or distal to the suture-button. This is a hybrid technique.

\section{Lateral fixation}

Three patients had lateral column Lisfranc's injuries (16\%) involving the fourth tarsometatarsal joint. Attention was directed to the dorsal aspect of the fourth tarsometatarsal joint. Layered dissection was made carefully to avoid any vital neurovascular structures. Sharp and blunt dissection was used to dissect through the extensor digitorum brevis muscle belly. After adequate exposure of the fourth tarsometatarsal joint, the joint was first stabilized with $\mathrm{k}$-wire fixation across the fourth tarsometatarsal joint, from distal to proximal and reduction was verified using fluoroscopic guidance. Then, suture anchor in the base of the fourth metatarsal and a knotless anchor was placed into the cuboid placing the suture from the anchor under tension. The suture was used to recreate the dorsal tarsometatarsal ligament with the joint held in anatomic reduction which may have better long-term stability (21). The calcaneal cuboid joint was also assessed for instability or subluxation. The k-wire was removed at the four-week post-operative appointment.

\section{RESULTS}

Nineteen patients were seen on average $19.4 \pm 30.1$ months post-index procedure for follow-up and evaluated by a Fellow not involved in the index surgery. These patients had their charts were evaluated at an average of $58.9 \pm 30.1$ months post-index surgery, by a different Fellow, again, not involved in the index surgery. All 19 patients had anatomic reductions noted immediately post-operatively. Their final post-operative weightbearing X-rays were noted to be anatomic in all patients taken at an average of $12.5 \pm 25.5$ months, though four patients were noted to have joint space narrowing at the time of that imaging $(21 \%)$. Of these four patients, three had medial injuries (two with screw and plate fixation, one with suture-button) and one with a lateral injury. Hardware removal was performed in six patients including one with a portion of their suture button. There were six patients with suture-button fixation, seven with metallic screws and plates, and six with bioabsorbable screws. Average RTA of the entire cohort was $24.0 \pm 10.3$ weeks (range $=10-50$ weeks). Medial Lisfranc's patients (16) had RTA $22.8 \pm 8.9$ weeks, while the three with lateral injuries $\mathrm{RTA}$ was $31.0 \pm 16.8$ weeks $(\mathrm{P}=0.21)$. Four patients returned to high level sports (three collegiately and one professionally), and none had decreased desired activity level. Post-op RM score for the cohort was $1.2 \pm 0.4$. There were no significant differences with the RTA, RM, post-op DJD in regard to sex, medial vs lateral injury, nor fixation type. The results are summarized in table I.

\section{DISCUSSION}

Lisfranc injuries are rare, complex and vary in their presentation and mechanism of injury. Low energy, indirect impact is typically the mechanism creating Lisfranc injury seen in the athletic population resulting in more subtle soft tissue injury. There is no consensus on the appropriate treatment, let alone the best type of fixation needed when surgical correction is required. MacMahon et al. showed that athletic patients had decreased participation or limitations to their activity after partial arthrodesis for Lisfranc injuries (22). MacMahon et al. reported on a cohort of 38 patients, average age of 31.8 years at the time of surgery, who engaged in high impact activities that had a limited primary arthrodesis. They concluded that these patients had decreased participation and increased difficulty with impact sports showing that many were experiencing limitations with high demand sports. There is also a concern for long term prognosis, especially in the athletic population due to the potential adverse incidence of osteoarthritis.

Porter et al. recent reported on a large series of competitive athletes. In 82 patients with an average age of 21 years (similar to our study) their cohort return to sport was 7.5 months. They did not perform any fusions in their patients nor report on arthritis (23).

Our study also showed that reduction was maintained at 1 year follow up, which is the most important indicator of outcome $(9,16-18,20)$. However, our series was too small to determine if loss of reduction or DJD was associated with fixation method. Despite metallic hardware having to be removed, that cohort had a faster RTA than other fixation types. The fact that the fixation type varied may be a sign the surgeon is paying attention to their results and following the patients.

Based on our current study findings, anatomic reduction, regardless of fixation used, showed to be an important predictor of outcome as suggested in several other studies $(9,17,24)$. Although Lisfranc injuries have been well documented and better recognized in the athletic population, their impact on their return on play is not always well documented $(25,26)$. McHale et al. showed that NFL ("American Football") players took an average of 11 months to return to competition; about $10 \%$ could not return to that level (27). Our study also demonstrated that surgical reduction and fixation without primary fusion leads to a successful return to activity as all our patients were able to return to their sporting activities. The patients in our study 
Table I. Subjects demographics and results.

\begin{tabular}{|c|c|c|c|c|c|c|c|c|c|}
\hline Injury & Side & Age & Sex & Procedure & Sport & Fixation & PO DJD? & HWR & RTA \\
\hline Fract/disloc & $\mathrm{R}$ & 18 & $\mathrm{~F}$ & ORIF Medial & Soccer & Bioabsorbable screw & No & No & 22.0 \\
\hline Dislocation & $\mathrm{L}$ & 17 & $\mathrm{~F}$ & ORIF Medial & Soccer & BIoabsorbable screw & No & No & 15.0 \\
\hline Sprain & $\mathrm{L}$ & 39 & $\mathrm{~F}$ & ORIF Lateral & Soccer & Bio-anchor and k-wire & Yes & No & 25.0 \\
\hline Sprain & $\mathrm{L}$ & 15 & F & ORIF Lateral & Swimming & K-wire & No & No & 18.0 \\
\hline Sprain & $\mathrm{L}$ & 15 & $\mathrm{~F}$ & ORIF Lateral & Volleyball & $\mathrm{K}$-wire & No & No & 50.0 \\
\hline Dislocation & $\mathrm{R}$ & 13 & $\mathrm{~F}$ & ORIF Medial & Soccer & K-wires & No & No & 18.0 \\
\hline Dislocation & $\mathrm{L}$ & 16 & $\mathrm{~F}$ & ORIF Medial & Runner & Plate and screws & Yes & Yes & 17.0 \\
\hline Dislocation & $\mathrm{R}$ & 20 & $\mathrm{~F}$ & ORIF Medial & Softball & Plate and screws & Yes & Yes & 15.0 \\
\hline Fracture & $\mathrm{R}$ & 30 & $\mathrm{~F}$ & ORIF Medial & Runner & Plate and screws & No & No & 30.0 \\
\hline Dislocation & $\mathrm{L}$ & 19 & $\mathrm{M}$ & ORIF Medial & Basketball & Plate and screws & No & Yes & 24.0 \\
\hline Dislocation & $\mathrm{R}$ & 38 & $\mathrm{M}$ & ORIF Medial & Basketball & Screw & No & Yes & 24.0 \\
\hline Sprain & $\mathrm{L}$ & 17 & $\mathrm{M}$ & ORIF Medial & Soccer & Screw & No & Yes & 15.0 \\
\hline Dislocation & $\mathrm{L}$ & 16 & $\mathrm{M}$ & ORIF Medial & Football & Screw & No & Yes & 23.0 \\
\hline Dislocation & $\mathrm{R}$ & 24 & $\mathrm{M}$ & ORIF Medial & Track Indoor & Endo-button & No & No & 31.0 \\
\hline Sprain & $\mathrm{R}$ & 30 & $\mathrm{M}$ & ORIF Medial & Runner & Endo-button & No & No & 48.0 \\
\hline Dislocation & $\mathrm{R}$ & 16 & $\mathrm{~F}$ & ORIF Medial & Soccer & Endo-button & No & No & 10.0 \\
\hline Sprain & $\mathrm{L}$ & 15 & $\mathrm{~F}$ & ORIF Medial & Runner & Endo-button & No & No & 21.0 \\
\hline Dislocation & $\mathrm{L}$ & 14 & $\mathrm{~F}$ & ORIF Medial & Soccer & Endo-button & No & No & 26.0 \\
\hline \multirow[t]{3}{*}{ Dislocation } & $\mathrm{R}$ & 19 & $\mathrm{~F}$ & ORIF Medial & Volleyball & Endo-button & Yes & No & 26.0 \\
\hline & & 20.6 & 6 & & & & 4 & & 22.8 \\
\hline & & 7.9 & 13 & & & & & & 9.4 \\
\hline
\end{tabular}

PO DJD?: Post-operative DJD noted at last visit; HWR: Hardware removal; RTA: Return to activity.

were able to resume their sport at an average of 24 weeks post-surgery, which was defined as beginning practice, not competition. The end point of return to competition may be highly variable and could be biased by when in the season an athlete sustained their injury. The RTA from our cohort is within similar time frames of previously reported $(5,23,25,26)$, with described time frames between 24 and 44 weeks $(25,27)$.

Other authors advocate primary arthrodesis for Lisfranc's injuries $(22,28,29)$. We did not compare this form of treatment and in general would not consider, unless the injury involved intra-articular, comminuted injury and patients of older age. The average age of our cohort was under 20 years. In the largest study on athletic patients $(\mathrm{n}=82)$, Porter $e t$ al. reported on a $50 \%$ incidence instance of inter-cuneiform ligament tears. Their cohort was of similar age as ours with an average return to sports of 7.5 months (30). This was a component of the injury we did not assess. Robertson et al. performed a systematic review and meta-analysis of 17 studies containing 366 athletes. They revealed percutaneous reduction, internal fixation (with a metal screw) had statistically better "return rates" and "mean return times" when compared to both open reduction internal fixation and primary arthrodesis (31).

As with any longitudinal clinical study, ours has weaknesses. The primary weakness was that fixation type varied. It was modified based on clinical experience and current literature at the time. For example, bioscrews were popular earlier in the series (primarily because they would not need to be removed), however over time, some of the screw heads worked their way subcutaneously and became prominent, necessitating removal. Also, the bioscrews could break across Lisfranc's junction and be symptomatic. Suture-button technique became popular around 2008 following the "success" of suture-buttons for ankle diastasis, the main benefit being the lack need of removal, however some of the buttons became prominent and also had to be removed. Regardless of the type of fixation used, our study showed a similar 
osteoarthritis development rate, seen in $21 \%$ of our subjects, coinciding with previously reported rates of $25 \%$ (9).

\section{CONCLUSIONS}

We can conclude from our study that ORIF regardless of fixation type for Lisfranc injury in athlete, is a safe and predictable treatment procedure if anatomical reduction is achieved. Faster RTA was achieved in those who had screw fixation and subsequent hardware removal. Based on this

\section{REFERENCES}

1. Desmond EA, Chou LB. Current concepts review: Lisfranc injuries. Foot Ankle Int 2006;27(8):653-60.

2. Cassinelli SJ, Moss LK, Lee DC, Phillips J, Harris TG. Delayed Open Reduction Internal Fixation of Missed, Low-Energy Lisfranc Injuries. Foot Ankle Int 2016;31(10):1084-90.

3. Ardoin GT, Anderson RB. Subtle Lisfranc injury. Tech Foot Ankle Surg 2010;9(3):100-6.

4. Lievers WB, Frimenko RE, Crandall JR, Kent RW, Park JS. Age, sex, casual and injury patterns in tarsometatarsal dislocations: a literature review of over 2000 cases. Foot (Edinb) 2012;22(3):117-24.

5. Lewis JS, Anderson RB. Lisfranc Injuries in the Athlete. Foot Ankle Int 2016;37(12):1374-80.

6. Eleftheriou KI, Rosenfeld PF. Lisfranc Injury in the Athlete. Foot Ankle Clin N Am 2013;18:219-36.

7. Eleftheriou KI, Rosenfeld PF. Lisfranc injury in the athlete: evidence supporting management from sprain to fracture dislocation. Foot Ankle Clin 2013;18(2):219-36.

8. Nunley JA, Vertullo CJ. Classification, investigation, and management of midfoot sprains: Lisfranc injuries in the athlete. Am J Sports Med 2002;30(6):871-8.

9. DeOrio M, Erickson M, Usuelli FG, Easley M. Lisfranc Injuries in Sport. Foot Ankle Clin N Am 2009;14(2):169-86.

10. Saxena, A. Lisfranc's Injuries. In: International Advances in Foot \& Ankle Surgery. (Saxena, editor). London: Springer 2011:pp. 229-234.

11. Raikin S, Elias I, Dheer S, Besser M, Morrison W, Zoga A. Prediction of midfoot instability in the subtle Lisfranc injury: Comparison of magnetic resonance imaging with intraoperative findings. J Bone Joint Surg Am 2009;91:892-9.

12. Jain K, Drampolos E, Clough T. Results of suture button fixation with targeting device aid for displaced ligamentous Lisfranc injuries in the elite athlete. Foot 2017;30:43-46.

13. Puna R, Tomlinson M. The role of percutaneous reduction and fixation of Lisfranc injuries. Foot Ankle Clin N Am 2017;22:15-34.

14. Saxena A, Bioabsorbable screws for reduction of Lisfranc's diastasis in athlete. Foot Ankle Surg 2005;44(6):445-9.

15. Koperen P, deJong V, Luitse J, Schepers T. Functional Outcomes after temporary bridging with locking plates in Lisfranc Injuries. J Foot Ankle Surg 2016;55:922-6.

16. Thordarson D, Hurvitz G. PLA Screw Fixation of Lisfranc Injuries. Foot Ankle Int 2002;23(11):1003-7.

17. Saxena A. Return to athletic activity after foot and ankle surgery: a preliminary report on select procedures. J Foot Ankle Surg 2000;39(2):114-9. experience, the senior author currently prefers this mode of fixation for medial diastasis injuries. Despite anatomic reduction, approximately $20 \%$ of athletes will have radiographic evidence of arthrosis approximately two-years post-surgery. Longer follow-up is needed to see if the arthrosis becomes limiting and requires more intervention.

\section{CONFLICT OF INTERESTS}

The authors declare that they have no conflict of interests.

18. Charlton T, Boe C, Thordarson D. Suture Button Fixation Treatment of Chronic Lisfranc Injury in Professional Dancers and High-Level Athletes. J Dance Med Sci 2015;19(4):135-9.

19. Saxena A, Hofer D. Stabilization of the Fourth Metatarsal-Cuboid Lateral Lisfranc Injury: Lateral Results of an Innovative Technique Using Suture Anchors. J Foot Ankle Surg 2018;57(2):409-13.

20. Nery C, Giza E, Wagner E, et al. Dynamic Lisfranc Joint Repair Concept: Surgical Technique for a Synthetic Neoligamentplasty. Muscles Ligaments Tendons J 2019;9(4):562-70.

21. Roles NC, Maudsley RH. Radial tunnel syndrome: resistant tennis elbow as nerve entrapment. J Bone Joint Surg 1972;54:499-508.

22. MacMahon A, Kim P, Levine D, et al. Return to sports and physical activities after primary partial arthrodesis for Lisfranc injuries in young patients. Foot Ankle Int 2016;37(4):355-62.

23. Porter D, Barnes A, Rund A, Walrod M. Injury pattern in ligamentous ligamentous injuries in competitive athletes. Foot Ankle Int 2018;40(2):185-94.

24. Rupesh AP, Matthew PWT. The role of percutaneous reduction and fixation of Lisfranc injuries. Foot Ankle Clin N Am 2017;22:15-34.

25. Deol R, Roche A, Calder J. Return to training and playing after acute Lisfranc injuries in elite professional soccer and rugby players. Am J Sports Med 2015;44(1):166-70.

26. Hong CC, Pearce CJ, Ballal MS, Calder JD. Management of sports injuries of the foot and ankle: An update. Bone Joint J 2016;98-B(10):1299-1311.

27. McHale K, Rozelle J, Milby A, Carey J, Sennett B. Outcomes of Lisfranc injuries in the national football league. Am J Sports Med 2016;44(7):1810-7.

28. Smith N, Stone C, Furey C. Does open reduction and internal fixation versus primary arthrodesis improve patient outcomes for Lisfranc trauma? A systematic review and meta-analysis. Clin Orthop Relat Res 2016;474:1445-52.

29. Weatherford B, Bohay D, Anderson J. Open reduction and Internal Fixation versus primary arthrodesis for Lisfranc Injuries. Foot Ankle Clin Am 2017;22:1-14.

30. Porter DA, Barnes AF, Rund A, Walrod MT. Injury Pattern in Ligamentous Lisfranc Injuries in Competitive Athletes. Foot Ankle Int 2019;40(2):185-94.

31. Robertson GAJ, Ang KK, Maffulli N, Keenan G, Wood AM. Return to sport following Lisfranc injuries: A systematic review and meta-analysis. Foot Ankle Surg 2019;25(5):654-64. 